\title{
Assessing Arthritis in the Temporomandibular Joint
}

A variety of characteristics make the temporomandibular joint (TMJ) distinct from other joints: (1) The bony connection with the contralateral TMJ, through the mandible, and the great variety of movement directions and trajectories make movements in this joint extremely complex and render an exact assessment of the range of motion of each single TMJ impossible; (2) the tight temporomandibular ligament, part of the lateral joint capsule, impedes the palpatory assessment of joint swelling or effusion; (3) the joint surface is covered with fibrous cartilage, below which immediately follows a zone of pluripotent proliferating cells promoting the growth of the whole mandible, the bone with the highest growth rate of the head; and (4) although pain from the temporomandibular region is not uncommon, it is highly unspecific and not a reliable indicator for TMJ arthritis ${ }^{1}$.

In this issue of The Journal, Stoll, et al present their study about intraarticular (IA) infliximab therapy for TMJ arthritis in children with juvenile idiopathic arthritis (JIA) ${ }^{2}$. Because of the lack of convincing treatment strategies for TMJ arthritis, especially in children, this report is of high interest. While for most other joints IA steroid injections are a recommended treatment option ${ }^{3}$, there is increasing awareness of severe negative effects of steroid injections into the TMJ, such as IA calcifications or ossifications or growth impairment of the mandibular ramus $s^{4,5,6}$. Finding an effective treatment alternative for this joint would certainly be intriguing. But the report of Stoll, et al has an additional feature that merits attention: Interestingly, the main focus of their report is not on the novel therapeutic idea, but on the magnetic resonance imaging (MRI) changes ${ }^{2}$.

\section{Why is MRI Assessment of Treatment Effect So Important?}

Most clinicians would probably state that the most important treatment goal is reduction of pain and discomfort for the patient, followed by improvement or restoration of function. Improvement of these 2 variables (clinical improvement) was
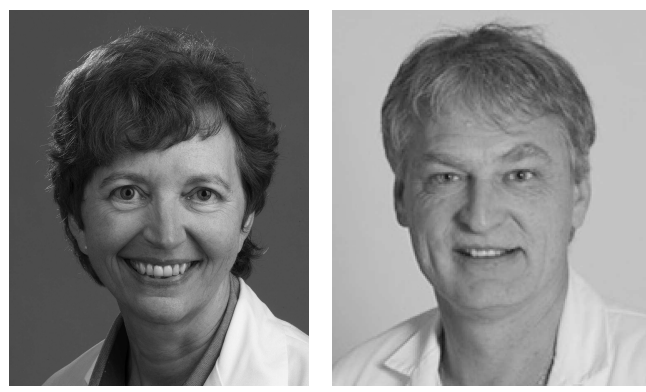

regarded as the first sign of decreasing disease activity, which would eventually also be visible on diagnostic imaging. However, looking back on the experience with TMJ injections, pediatric rheumatologists have learned that clinical assessment may be misleading. Although the consequences of untreated TMJ arthritis have been well studied already for decades, it was only after MRI became a common diagnostic method that it was recognized how often and how early in the course of JIA TMJ involvement takes place ${ }^{7,8,9}$. Many efforts aimed to find clinical tools that reliably predicted early TMJ arthritis before secondary growth disturbance and bony destruction had taken place. Unfortunately, neither method - examination by experienced clinician or ultrasound - was found to have adequate sensitivity or specificity for this goal ${ }^{1,8}$.

While radiological methods (radiographs, computerized tomography, digital volume tomography) demonstrate well the shortening of the mandibular ramus and deformity of the TMJ, mainly consisting of flattening and erosive changes of the mandibular condyle, MRI is the only method to reliably show imaging signs of acute inflammation.

Synovial inflammation is characterized on MRI by thickening of the synovium (hypertrophy, hyperplasia), joint effusion, and increased joint enhancement due to hyperemia, as well as increased extravasation and diffusion of gadolinium-based contrast agents into the joint space (Figure 1). Increased joint enhancement has been shown to correlate with the presence of inflammatory activity on histology ${ }^{10}$.

However, there is still some uncertainty about what should be considered an early sign of inflammation. Although some investigators regard any intraarticular fluid and any contrast enhancement indicative of inflammation ${ }^{7,11,12}$, we and others have found small amounts of intraarticular fluid as well as joint enhancement within minutes of contrast application in TMJ of children without TMJ pathology ${ }^{13,14}$. Such discrepant appreciation of the normal amounts of synovial fluid and normal degree of joint

See IA IFX for TMJ in RA, page 2155

Personal non-commercial use only. The Journal of Rheumatology Copyright $@$ 2015. All rights reserved 


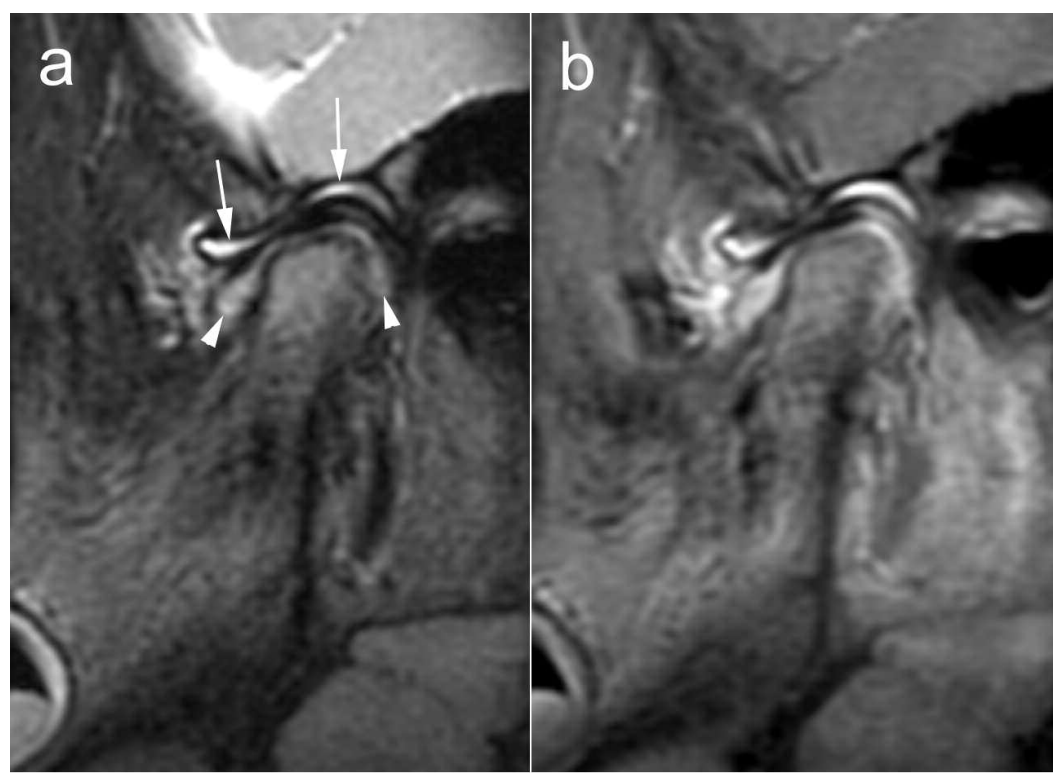

Figure 1. MRI of TMJ involvement in a 3-year-old boy with JIA. Fat-saturated T2-weighted image (a) shows small effusion (arrows) in upper joint compartment and thickened synovium (arrowheads) in lower joint compartment. Both effusion and thickened synovium show strong enhancement on fat-saturated T1-weighted image (b) following intravenous contrast administration. Note mild deformity of the TMJ with flattening of the mandibular condyle. MRI: magnetic resonance imaging; TMJ: temporomandibular joint; JIA: juvenile idiopathic arthritis

enhancement is most likely explained by the use of diverse MRI machines, coils, sequences, and imaging planes for assessing TMJ at different centers. Therefore all investigators should be aware of the normal appearance of TMJ on their own machine and with their own imaging protocol before TMJ inflammation can be diagnosed and its severity graded. So far, every center, including the Birmingham Alabama group in the report of Stoll, et al, has developed and used its own scoring system for assessing the intensity of inflammation and treatment effect ${ }^{2,5,15}$. Although all scoring systems distinguish between acute inflammatory and more chronic deformative or destructive osteochondral changes, definition of imaging findings and their grading varies. Currently the OMERACT working group MRI in JIA is evaluating existing scoring systems ${ }^{16,17,18}$ and developing a consensus for defining and grading of inflammatory MRI findings in TMJ. Such a consensus on the MRI protocol and scoring of inflammatory TMJ findings will in future allow comparing the effects of the different therapies performed at different centers. For assessing the effect of systemic or specific treatments targeting the TMJ, evaluation of the level of inflammation, the degree and course of bony deformation, as well as growth of the mandibular ramus in the long term is needed.

MRI seems to remain the only method able to detect early TMJ arthritis. And if the diagnosis of TMJ arthritis can reliably be made only by using MRI, then a scientific measurement of the response to treatment would need to be done using the same method. At the same time, MRI can also provide longterm outcome measures such as progression or improvement of osteochondral TMJ deformities and growth of the mandibular ramus. MRI has been shown to be as accurate as radiological methods for measuring the height of the mandibular ramus ${ }^{19}$.

IA infliximab with the dose and frequency used did not improve TMJ arthritis in this study. However, by using an MRI-based assessment for measuring the effect of their novel treatment, Stoll, et al have set new standards with their work.

ROTRAUD K. SAURENMANN, MD,

Division of Rheumatology,

Zurich University Children's Hospital; and

Department of Pediatrics,

Cantonal Hospital Winterthur, Winterthur;

CHRISTIAN J. KELLENBERGER, MD,

Diagnostic Imaging,

Zurich University Children's Hospital,

Zurich, Switzerland.

Address correspondence to Dr. R.K. Saurenmann, Department of Rheumatology, University Children's Hospital, Steinwiesstr. 75, Zurich,

Switzerland, CH-8032; E-mail: traudel.saurenmann@kispi.uzh.ch

\section{REFERENCES}

1. Muller L, Kellenberger CJ, Cannizzaro E, Ettlin D, Schraner T, Bolt IB, et al. Early diagnosis of temporomandibular joint involvement in juvenile idiopathic arthritis: a pilot study comparing clinical examination and ultrasound to magnetic resonance imaging. Rheumatology 2009;48:680-5.

2. Stoll ML, Vaid YN, Guleria S, Beukelman T, Waite PD, Cron RQ. Magnetic resonance imaging findings following intraarticular infliximab therapy for refractory temporomandibular joint arthritis among children with juvenile idiopathic arthritis. J Rheumatol 2015;42:2155-9.

Personal non-commercial use only. The Journal of Rheumatology Copyright (c) 2015. All rights reserved. 
3. Beukelman T, Patkar NM, Saag KG, Tolleson-Rinehart S, Cron RQ, DeWitt EM, et al. 2011 American College of Rheumatology recommendations for the treatment of juvenile idiopathic arthritis: initiation and safety monitoring of therapeutic agents for the treatment of arthritis and systemic features. Arthritis Care Res 2011;63:465-82.

4. Ringold S, Thapa M, Shaw EA, Wallace CA. Heterotopic ossification of the temporomandibular joint in juvenile idiopathic arthritis. J Rheumatol 2011;38:1423-8.

5. Lochbuhler N, Saurenmann RK, Muller L, Kellenberger CJ. Magnetic resonance imaging assessment of temporomandibular joint involvement and mandibular growth following corticosteroid injection in juvenile idiopathic arthritis. J Rheumatol 2015; 42:1514-22.

6. Hugle B, Laxer RM. Lipoatrophy resulting from steroid injection into the temporomandibular joint. Arthritis Rheum 2009;60:3512.

7. Kuseler A, Pedersen TK, Herlin T, Gelineck J. Contrast enhanced magnetic resonance imaging as a method to diagnose early inflammatory changes in the temporomandibular joint in children with juvenile chronic arthritis. J Rheumatol 1998;25:1406-12.

8. Weiss PF, Arabshahi B, Johnson A, Bilaniuk LT, Zarnow D, Cahill $\mathrm{AM}$, et al. High prevalence of temporomandibular joint arthritis at disease onset in children with juvenile idiopathic arthritis, as detected by magnetic resonance imaging but not by ultrasound Arthritis Rheum 2008;58:1189-96.

9. Kuseler A, Pedersen TK, Gelineck J, Herlin T. A 2 year followup study of enhanced magnetic resonance imaging and clinical examination of the temporomandibular joint in children with juvenile idiopathic arthritis. J Rheumatol 2005;32:162-9.

10. Kuseler A, Pedersen TK, Barlach J, Gelineck J, Sangill R, Melsen $\mathrm{B}$, et al. Contrast-enhanced MRI compared to histological findings in the temporomandibular joint of antigen-induced arthritis in young rabbits. Clin Exp Rheumatol 2004;22:441-6.
11. Tzaribachev N, Fritz J, Horger M. Spectrum of magnetic resonance imaging appearances of juvenile temporomandibular joints (TMJ) in non-rheumatic children. Acta Radiol 2009;50:1182-6.

12. Stoll ML, Sharpe T, Beukelman T, Good J, Young D, Cron RQ. Risk factors for temporomandibular joint arthritis in children with juvenile idiopathic arthritis. J Rheumatol 2012;39:1880-7.

13. Kottke R, Saurenmann RK, Schneider MM, Muller L, Grotzer MA, Kellenberger CJ. Contrast-enhanced MRI of the temporomandibular joint: findings in children without juvenile idiopathic arthritis. Acta Radiol 2015;56:1145-52.

14. von Kalle T, Winkler P, Stuber T. Contrast-enhanced MRI of normal temporomandibular joints in children -is there enhancement or not? Rheumatology 2013;52:363-7.

15. Cahill AM, Baskin KM, Kaye RD, Arabshahi B, Cron RQ, Dewitt EM, et al. CT-guided percutaneous steroid injection for management of inflammatory arthropathy of the temporomandibular joint in children. AJR Am J Roentgenol 2007;188:182-6.

16. Kellenberger CJ, Arvidsson LZ, Larheim TA. Magnetic resonance imaging of temporomandibular joints in juvenile idiopathic arthritis. Semin Orthodont 2015;21:111-20.

17. Koos B, Tzaribachev N, Bott S, Ciesielski R, Godt A. Classification of temporomandibular joint erosion, arthritis, and inflammation in patients with juvenile idiopathic arthritis. J Orofac Orthop 2013;74:506-19.

18. Vaid YN, Dunnavant FD, Royal SA, Beukelman T, Stoll ML, Cron $\mathrm{RQ}$. Imaging of the temporomandibular joint in juvenile idiopathic arthritis. Arthritis Care Res 2014;66:47-54.

19. Markic G, Muller L, Patcas R, Roos M, Lochbuhler N, Peltomaki T, et al. Assessing the length of the mandibular ramus and the condylar process: a comparison of OPG, CBCT, CT, MRI, and lateral cephalometric measurements. Eur J Orthod 2015;37:13-21.

J Rheumatol 2015;42:2000-2; doi:10.3899/jrheum.151088 